\title{
Persuasive Health: Back to the Future
}

\author{
Emma D. Wilson \\ Sumi Helal \\ Christopher N. Bull \\ Mahsa Honary \\ e.d.wilson1@lancaster.ac.uk \\ Centre on Digital Health and Quality of Life Technologies, School of Computing and Communications, Lancaster \\ University, Lancaster, UK
}

\begin{abstract}
With individual behaviour and lifestyle determining 30-50\% of people's health, research and supportive technology for affecting behaviour alteration remain urgently needed. Most existing persuasive systems are designed to persuade a user to change a finite set of behaviours to achieve a specific goal. However, if the user's situation or goal changes, such systems cannot adapt to the changes. A much more robust type of persuasive system is needed today to enable adequate health navigation and to empower people to face and change their own realities in terms of a large variety of health behaviours and lifestyles. In this paper, we provide a perspective on the impressive body of work contributed over the past 15 years, to better look into the future of persuasive health and to the opportunities a broader theoretical framework and practical methodologies may bring about. We present a taxonomy that attempts to explain the contributions in this field including health behaviour theory, cybernetic actionbehaviour models, social cognitive theory, and control theory. We identify potentially promising approaches to advance persuasive health's efficacy in empowering individuals to improve their own health outcomes.
\end{abstract}

\section{CCS CONCEPTS}

- Human-centered computing $\rightarrow$ User models; HCI theory, concepts and models; Interaction design theory, concepts and paradigms; • Computing methodologies

Permission to make digital or hard copies of all or part of this work for personal or classroom use is granted without fee provided that copies are not made or distributed for profit or commercial advantage and that copies bear this notice and the full citation on the first page. Copyrights for components of this work owned by others than ACM must be honored. Abstracting with credit is permitted. To copy otherwise, or republish, to post on servers or to redistribute to lists, requires prior specific permission and/or a fee. Request permissions from permissions@acm.org.

PervasiveHealth'19, May 20-23, 2019, Trento, Italy

(C) 2019 Association for Computing Machinery.

ACM ISBN 978-1-4503-6126-2/19/05 . .\$15.00

https://doi.org/10.1145/3329189.3329245 $\rightarrow$ Computational control theory; • Applied computing $\rightarrow$ Psychology; Sociology; Consumer health; Health informatics; • Mathematics of computing $\rightarrow$ Differential equations.

\section{KEYWORDS}

Persuasive health, behaviour theory, patient empowerment, e-coaching, human actuation, human in the loop, control theory

\section{ACM Reference Format:}

Emma D. Wilson, Sumi Helal, Christopher N. Bull, and Mahsa Honary. 2019. Persuasive Health: Back to the Future. In The 13th International Conference on Pervasive Computing Technologies for Healthcare (PervasiveHealth'19), May 20-23, 2019, Trento, Italy. ACM, New York, NY, USA, 9 pages. https://doi.org/10.1145/3329189.3329245

\section{INTRODUCTION}

Health behaviours such as smoking, physical inactivity and poor diet are major risk factors for a number of chronic diseases including heart disease, type 2 diabetes, and some cancers [56]. An unhealthy diet and a lack of physical activity are the leading causes of avoidable illness and premature death in Europe [45]. Furthermore, over 30-50\% of an individual's health is determined by lifestyle and health behaviour [5]. Despite this evidence, sedentary behaviour and unhealthy eating habits are established in many societies. In a survey of British adults, $76 \%$ did not eat enough fruit and vegetables and 66\% lack physical exercise [53]. There is a need to motivate health behaviour change in order to improve health.

Digital platforms such as smartwatches, wearables and smartphones provide platforms that can be utilised to persuade health behaviour change. They incorporate sensing capabilities via accelerometers, GPS, heart rate monitors and microphones and can be used to provide timely support as well as monitoring in real-time [6, 21, 33]. Advantages of these platforms include their availability and accessibility, cost effective delivery, scalability, ability to personalise content and the capability of providing real-time strategies $[6,21]$. 
We have the platforms to support health behaviour change [23]. Indeed, reviews of the literature indicate that persuasive technologies do seem to persuade people into various behaviours $[17,37]$ (although more work is required to establish their long term effect). What is lacking is standardisation in both the methods used to evaluate effectiveness and coherent theories to improve behaviours [17, 32, 37, 39, 56]. A lack of theory limits our ability to better understand possible mechanisms of change and further improve behaviours. It also makes it hard to answer how to adapt to changing needs and individual behaviours. Theories are necessary to effectively personalise digital health interventions [18] and make use of the personal digital platforms that smartphones and wearables provide us with.

The problem may be that current models and theories of behaviour are inadequate for the needs of persuasive technologies. Most current models and theories of behaviour change do not capture how behaviour changes over time, making it hard to use them in digital health interventions [18, 19, 32, 41, 45, 52]. In using existing models we are effectively trying to use a static model in a dynamic world. There is growing recognition of the need to develop computational models of behaviour that quantify interactions between states and how these relationships vary over time $[15,18,41,42]$.

In addition to a need for dynamic behavioural models, there is a need to develop decision rules that use these models to make decisions that improve behavioural outcomes. Control systems engineering (control theory) is concerned with the behaviour of dynamical systems and the design of closed loop systems. It provides a systematic way of determining inputs in the presence of noise and uncertainty. Techniques from control theory are increasingly being used for interdisciplinary applications such as climate control, economics, and for medical treatments [7, 29, 47, 57]. It has been suggested that methods from control theory could help determine rules for adaptive digital interventions [3, 10$12,30,42-44,55]$.

In this contribution, we review existing behavioural change theories and look at how these can and have been used to develop dynamic models of behaviour change. We describe how control theory could provide the theoretical framework that is currently lacking from persuasive technologies, highlighting where these techniques have been used in the literature as well as future research challenges. We hope to provide some ideas of where we need to go next and how we might get there in order to improve persuasion in persuasive health.

\section{BEHAVIOURAL CHANGE THEORIES}

There is a vast body of work within the social sciences on behavioural change. This is exemplified by the book ABC of behavioural change theories, which describes 83 models of behavioural change [31]. Key behavioural change models include the health belief model (HBM) [2], the health action process approach (HAPA) [46], social cognitive theory (SCT) [1], and the transtheoretical model (TTM) [40]. These models provide an overview of the main pathways thought to drive behaviour change [9]. Key themes from behavioural models are that outcome expectations, risk perception, goals, selfefficacy, impediments and planning can influence health behaviour and cues to actions can activate health behaviour when appropriate beliefs are held.

There is a need to incorporate theories for behavioural change in developing more effective persuasive digital health interventions $[22,32]$. In practice few digital interventions delivered via mobile applications provide a theoretical basis to the behaviour change [39]. Even where a theoretical model is stated it can be hard to see how exactly the theory maps onto the application $[32,56]$. It is rare that hypothesised theoretical connections are investigated systematically [56]. With this disconnect between theory and practice, it is difficult to disentangle where to attribute improvements in behaviour. This makes it hard to develop methods to further improve behaviours and does not help to answer how to adapt to changing or individual requirements.

An action based behavioural model (ABM) was developed to address some of the barriers in utilising behavioural theory in persuasive technologies [27, 28]. ABM was developed to: i) provide a behaviour change model, based on collective knowledge gained from social and behavioural science theories, within a framework accessible to developers and computer scientists, and ii) as a solution to the problem that most current persuasive systems are designed to change a finite set of behaviours and cannot adapt if the users situations and goals change. The ABM model is proposed as a persuasion template, it partitions the telehealth system into a cyber system and set of user actions (see Fig 1.). From 'Start', the user steps through a set of actions from 'Aware' to 'Act'. Cyber Influences affect and help the user take each action and Cyber Senses monitor the user actions. After acting, the Cyber Action 'Assess' evaluates the achievements and advises the user on the next action. To measure compliance a Situation-based Assess Tree (SAT) was developed alongside the ABM [26]. This enabled the assessment of user behaviour responsiveness and compliance to cyber influence.

ABM and SAT attempt to provide a framework for addressing the limitation that current behavioural models are difficult for computer scientists and developers to interpret and it is difficult to adapt to changing situations and goals. However, this approach does not question why such models are so difficult to interpret and use. The difficulty arises as we are attempting to apply steady-state, static behavioural models to a dynamic world [19, 32, 41, 45, 51, 52]. Most current models and theories of behaviour change do not capture 


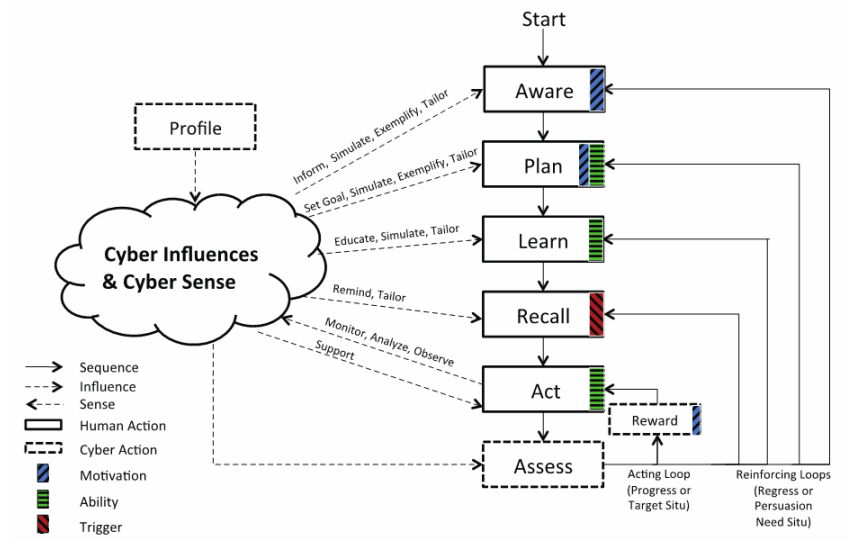

Figure 1: An action based behaviour model, from [26].

how behaviour changes over time, making it hard to use them in digital health interventions. We now need to build on existing theories to develop computational models of behaviour that not only specify relationships, but quantify how relationships vary over time and in context, and then use these to develop digital health interventions with a strong theoretical basis.

\section{DYMANIC MODELS OF BEHAVIOUR CHANGE}

Historically, data collection methods such as face to face interviews or paper questionnaires have only enabled sparse collection of results. This has led to behavioural theories that do not include time and simply provide a steady state snapshot of factors that affect behaviours. Advantages of using computers in persuasive systems include the fact they can store large amounts of data and can be ubiquitous [14] These advantages need to be exploited to obtain intensive longitudinal data that can be used to inform new dynamic behavioural models. The use of Ecological momentary assessment (EMA) on mobile devices [48] and utilisation of signals from wearables provide examples of how we can obtain the necessary intensive longitudinal data to develop dynamic models of behaviour change $[18,23]$.

\section{Simple dynamic example model}

In SCT self-efficacy is the key driver for behaviour change. Bandura [1] identified four factors that influence SCT: mastery experiences, vicarious experiences, verbal persuasion and emotional and physiological states. We first motivate the need for dynamic models of behaviour by using a simple model of self-efficacy in response to mastery, guided by principles from Bandura's SCT. A very simple linear dynamic model of how mastery may influence self-efficacy in open-loop (the effect of feedback is not included) is,

$$
\dot{x}_{S E}=k u-\frac{1}{\tau} x_{S E}
$$

where $x_{S E}$ is self-efficacy, $\dot{x}_{S E}$ is the first derivative of selfefficacy w.r.t time, $u$ is mastery, and $k$ and $\tau$ model parameters that describe the gain and time constant respectively. This demonstrates how a dynamic model of behaviour not only defines the model structure (e.g., the idea that the mastery drives self-efficacy), but also the magnitude and time-course of response.

Simulated results using this model with $\tau=5$ and $k=1$ are shown in Fig. 2. The simple model is able to capture several phenomena: i) if mastery is short lived then selfefficiency decays back to a baseline value, ii) if the experience is negative then self-efficacy is also negative, iii) if mastery is constant then the increase in self efficacy is greatest initially and then reaches a steady state.

This example is intended to show how dynamic models can provide rich predictions of how behaviour evolves over time. To incorporate other more complex factors thought to affect self-efficacy, such as the idea that self-efficacy is less likely to be affected by a negative experience if prior to the experience we have had a lot of positive experiences [1], non-linearities could be included. Individual effects, such as the idea that some individuals have higher self-efficacy that is harder to deplete, could be incorporated by varying the model parameters.

\section{State of the art}

There have been some attempts to develop dynamic models of behaviour. However, in comparison to work within the social sciences on behavioural change theories, this field remains in its infancy. Dynamical models of smoking cessation [4], alcohol consumption [24] and physical activity in response to text message micro-interventions $[49,50]$ have been developed.

The first attempts at developing a general behaviour model have been made by Rivera et al. [30, 34, 43]. Early versions of their dynamic behaviour model are based on the theory of planned behaviour (TBP) [34], with later versions based on SCT [30]. Their models have been applied to smoking cessation [55], fibromyalgia pain [10], weight gain [13, 34], and to physical activity [43]. Thus far the examples have been quite simple with inputs and outputs that can easily be quantified such as the number of steps walked [43].

The dynamical SCT model can be represented using a state-space representation, where the system is modelled as a set of input, output and state variables related via first order differential equations. State-space models are widely used in Control Systems Engineering and can be described in general as 

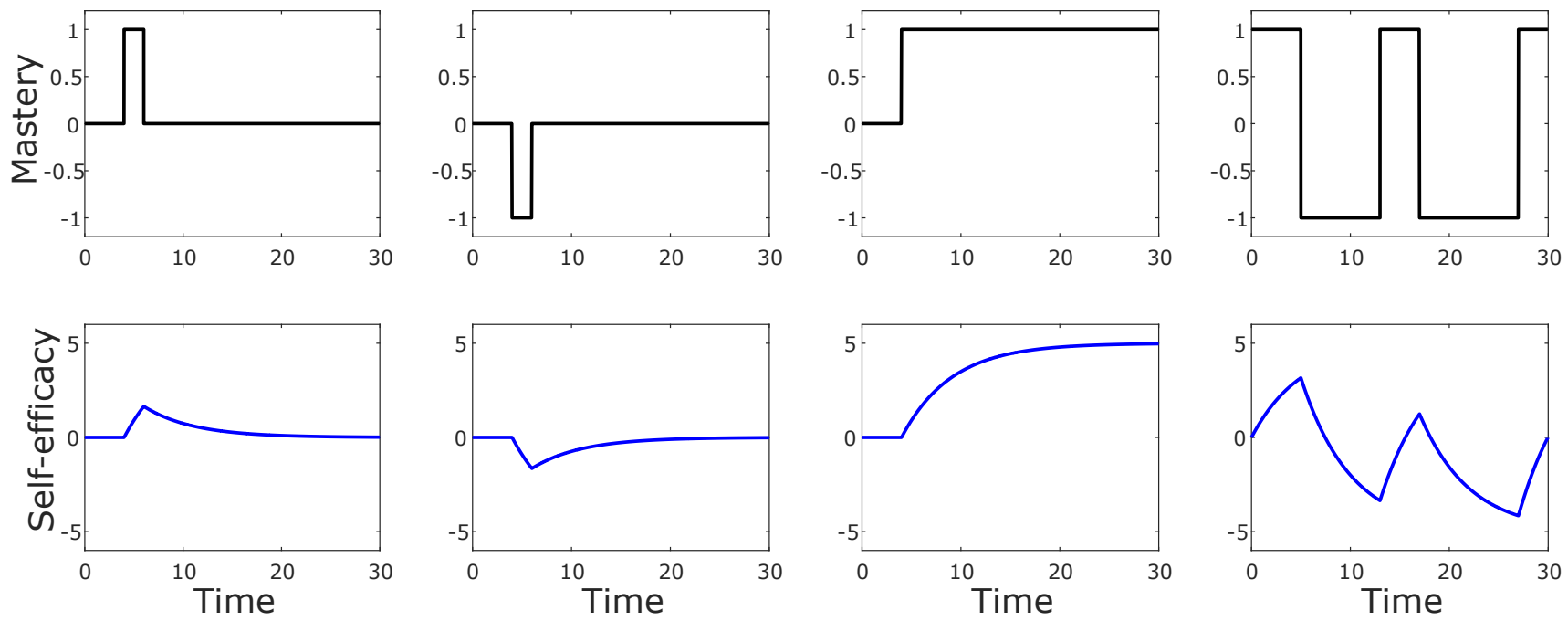

Figure 2: The effect of mastery on self efficacy. Top plots show mastery inputs and bottom plots the corresponding self-efficacy, simulated using Eq. (1).

$$
\begin{aligned}
& \dot{x}=A x+B u \\
& y=C x+D u
\end{aligned}
$$

where $\boldsymbol{x}$ is a vector of states, $\boldsymbol{u}$ the inputs and $\boldsymbol{y}$ the outputs. In the dynamic SCT model there are eight inputs: skills training $\left(\varsigma_{1}\right)$, observed behaviour $\left(\varsigma_{2}\right)$, perceived social support and verbal persuasion $\left(\varsigma_{3}\right)$, internal cues $\left(\varsigma_{4}\right)$, perceived barriers and obstacles $\left(\varsigma_{5}\right)$, intrapersonal states $\left(\varsigma_{6}\right)$, environmental context $\left(\varsigma_{7}\right)$, external cues $\left(\varsigma_{8}\right)$. There are six states: self management skills $\left(\eta_{1}\right)$, outcome expectancies $\left(\eta_{2}\right)$, selfefficacy $\left(\eta_{3}\right)$, behaviour $\left(\eta_{4}\right)$, behavioural outcomes $\left(\eta_{5}\right)$, cue to action $\left(\eta_{6}\right)$. The outcomes of interest are often the behaviour and self-efficacy. In mapping the SCT model onto a state-space representation the state matrix,

$\boldsymbol{x}=\left[\begin{array}{llllll}\eta_{1} & \eta_{2} & \eta_{3} & \eta_{4} & \eta_{5} & \eta_{6}\end{array}\right]^{\prime}$ is a vector of $n=6$ states, and the input matrix $\boldsymbol{u}=\left[\begin{array}{llllllll}\varsigma_{1} & \varsigma_{2} & \varsigma_{3} & \varsigma_{4} & \varsigma_{5} & \varsigma_{6} & \varsigma_{7} & \varsigma_{8}\end{array}\right]^{\prime}$ a vector of $m=8$ input variables. If the outputs of interest are self-efficacy and behaviour, then $y=\left[\begin{array}{ll}\eta_{3} & \eta_{4}\end{array}\right]^{\prime}$ is a vector of $p=2$ output variables. The matrixes $A, B$ and $C$ are state matrices which contain the model parameters,

$$
A=\left[\begin{array}{cccccc}
-\frac{1}{\tau_{1}} & 0 & 0 & \frac{\beta_{14}}{\tau_{1}} & 0 & 0 \\
\frac{\beta_{21}}{\tau_{2}} & -\frac{1}{\tau_{1}} & 0 & \frac{\beta_{25}}{\tau_{2}} & 0 & 0 \\
\frac{\beta_{31}}{\tau_{3}} & 0 & -\frac{1}{\tau_{3}} & \frac{\beta_{34}}{\tau_{3}} & 0 & 0 \\
0 & \frac{\beta_{42}}{\tau_{4}} & \frac{\beta_{43}}{\tau_{4}} & -\frac{1}{\tau_{4}} & \frac{\beta_{45}}{\tau_{4}} & \frac{\beta_{46}}{\tau_{4}} \\
0 & 0 & 0 & \frac{\beta_{54}}{\tau_{4}} & -\frac{1}{\tau_{5}} & 0 \\
0 & 0 & 0 & 0 & 0 & -\frac{1}{\tau_{4}}
\end{array}\right]
$$

$$
\begin{gathered}
\boldsymbol{B}=\left[\begin{array}{cccccccc}
\frac{\gamma_{11}+1}{\tau_{1}} & 0 & 0 & 0 & 0 & 0 & 0 & 0 \\
0 & \frac{\gamma_{22}+1}{\tau_{1}} & 0 & 0 & 0 & 0 & 0 & 0 \\
0 & \frac{\gamma_{32}}{\tau_{3}} & 0 & 0 & -\frac{\gamma_{35}}{\tau_{3}} & \frac{\gamma_{36}}{\tau_{3}} & 0 & 0 \\
0 & 0 & 0 & \frac{1}{\tau_{4}} & 0 & 0 & 0 & 0 \\
0 & 0 & 0 & 0 & \frac{1}{\tau_{5}} & 0 & \frac{\gamma_{57}}{\tau_{5}} & 0 \\
0 & 0 & 0 & \frac{\gamma_{64}}{\tau_{6}} & 0 & \frac{1}{\tau_{6}} & 0 & \frac{\gamma_{66}}{\tau_{6}}
\end{array}\right] \\
C=\left[\begin{array}{cccccc}
0 & 0 & 1 & 0 & 0 & 0 \\
0 & 0 & 0 & 1 & 0 & 0
\end{array}\right]
\end{gathered}
$$

where $\beta, \gamma$ and $\tau$ are used to define the model parameters. In practice when the model has been applied, it has been simplified to consider fewer inputs. For example, in [30] a two input model was considered, with skills training and external cues chosen as the inputs which were measured by the time spent reading tips and the number of reminders sent to set a new goal respectively. In this case the outcomes of interest were the behaviour and self-efficacy.

Unknown model parameters for human behaviour models have been identified using system identification techniques $[15,30,42]$. System identification involves testing the response of a system to different inputs over time and using this data to estimate model parameters, or refine models. It is key to ensure that the inputs used can excite the system dynamics adequately to ensure we are able to capture the relevant range of dynamics. In applying these techniques to estimate a dynamic human behaviour model we are limited in how we can vary inputs. We can only really change inputs using push factors (e.g., via cues to action, reminders, or prompts) [23], which can add burden to the user. In order to measure some of the internal states, for example outcome expectancies, EMAs can be used [23]. Again, this can be 
burdensome to the individual. Careful thoughts needs to be made to determine what exactly our data sets should contain, for example we could include metrics on the environment, the weather, how busy the person is, among other elements. We have the capabilities to make these rich data sets [23], but these data sets still do not exist. Creating them requires very careful experimental design to ensure we can measure meaningful data.

\section{Modelling considerations}

Using the two input model and illustrative parameters defined in [30] $\left(\tau_{1}=1, \tau_{2}=1, \tau_{3}=1, \tau_{4}=2, \tau_{5}=1, \tau_{6}=\right.$ $3, \gamma_{11}=3, \gamma_{22}=1, \gamma_{32}=2, \gamma_{33}=1, \gamma_{35}=1, \gamma_{36}=1, \gamma_{57}=$ $2, \gamma_{64}=15, \gamma_{68}=15, \beta_{21}=0.3, \beta_{31}=0.5, \beta_{42}=0.3, \beta_{43}=$ $0.8, \beta_{45}=0.1, \beta_{54}=0.3, \beta_{24}=0.2, \beta_{25}=0.3, \beta_{14}=0.23, \beta_{46}=$ $0.44)$ the effect of external cues on behaviour were simulated to again demonstrate the importance of time. In the simulation, a period of 20 days was considered and for each day there was a random probability of one to five cues to action occurring randomly throughout the time period $8 \mathrm{am}$ till $8 \mathrm{pm}$. A typical input where the cues to action are represented by spikes at the times they occur is shown in Fig. 3a. The cues to action could also be represented using the total number of cues that occur on a typical day (Fig. 3b), the two inputs produce very similar responses (Fig. 3c). An additional input, busyness was next considered, we might expect that cues to action that occur when someone is busy have no effect. For illustrative purposes, a very simple input was used for busyness which was equal to one when someone was busy which was taken to be between 9am till 5pm on weekdays (Fig. 3d). By representing the input cues to action as spikes we are able to exclude the effect of any cues to action that occur when busy, giving the behaviour seen in Fig. 3d. We are unable to do this when the input is represented as the total cues for the day. This demonstrates not only the importance of timing, but how we represent temporal information. Careful thought needs to be given to what suitable sampling frequencies for collecting data and modelling are.

The SCT dynamical model of behaviour change provides a first step in developing a dynamic behaviour models. The model is based on linear dynamic systems theory. Better models, describing more complex behaviours, might be developed using models with state dependent parameters. In these models the state of the system effects the model parameters [58]. This links to the idea of teachable moments, that in certain states people are more likely to enact behavioural change [25]. From a dynamic systems modelling perspective the gain could be modelled as a function of states to be very high under conditions (certain configurations of states) that are considered to be teachable moments.

When modelling there is always a trade-off between model complexity and accuracy, any model should be parsimonious a)

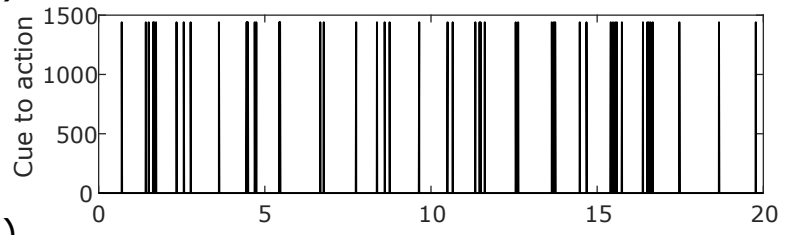

b)

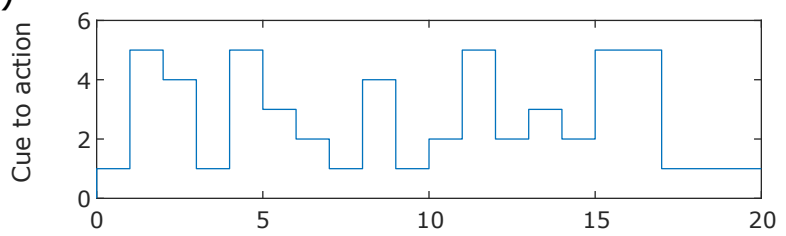

c)

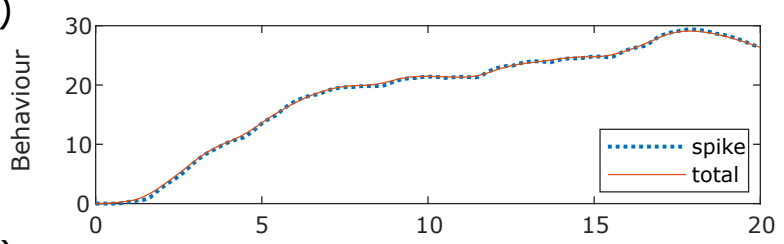

d)

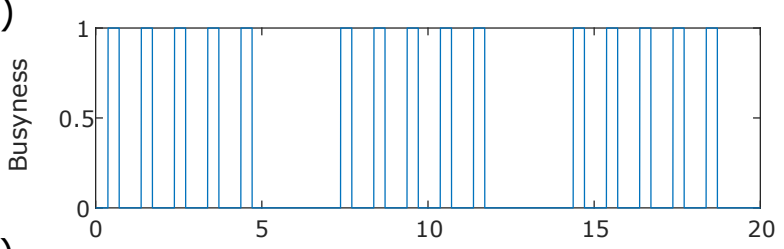

e)

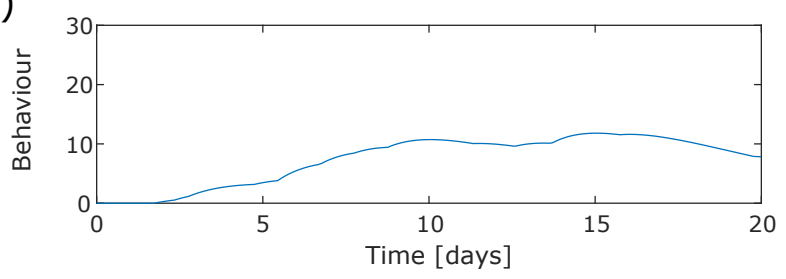

Figure 3: The importance of timing. a) Cues to action as spike inputs, b) Cues to action as totals per day, c) Behaviour when using both spikes and totals per day as inputs, d) Busyness, e) Behaviour when busyness taken into account.

and capture the key dynamics of the system being modelled. There are questions as to what complexity should be used in developing dynamic models of behaviour. To answer this and develop better dynamic models of behaviour we first need better data sets which include model inputs, outputs and state variables over time. Key questions in relation to behaviour change models are what states, inputs and outputs should be included in the models and over what time and how frequently do we need to sample.

\section{SYSTEMS AND CONTROL THEORY FOR PERSUASION}

In the previous sections we have discussed the need for better computational models of behaviour change for persuasive 
health, we now consider how these models could be used to make better decisions. We argue that control theory may be useful in developing theories for how to persuade behaviour change and describe some attempts in the literature where control theory is being used to determine digital interventions.

Control theory deals with the response of a dynamic system (often referred to as the 'plant') over time. It is concerned with designing control laws (referred to as 'controllers') that will cause the system to respond in a desired manner [20]. The main concern is to ensure the controlled system behaves well in the presence of model uncertainty, external disturbances and noise. Control systems are prolific within modern industries and technology, with applications including aircraft control, temperature regulation, and robotics [20]. There are several biological functions which use feedback control and control methodologies are increasingly being used in non-standard applications [57]. These non-standard applications include climate control [29], biological systems [54], economics [47], resource management [35] and medical treatments [7].

Where control theory has been used for non-standard applications, controllers have been based either on proportionalintegral-derivative (PID) feedback control (Fig. 4), or model predictive control (MPC) [57]. In PID control, a feedback loop is used to feed back the output of the system. This is compared to a desired reference signal to give an error value $\left(e(t)=y_{d}(t)-y(t)\right)$ where $y_{d}(t)$ is the desired output and $y(t)$ the actual output). The control signal is a sum of three terms: one proportional to the error $(\mathrm{P})$, one proportional to the integral of the error (I) and one proportional to the derivative of the error (D) [20]. MPC is a model based control method that provides a method for optimising the performance of constrained systems. At each time instant a MPC: takes a measurement of the system state, computes a finite horizon control sequence that uses an internal model to predict system behaviour, minimises some cost function, and doesn't violate any constraints. The first values of the control sequence are then used as the next inputs to the system and the process is repeated each time step.

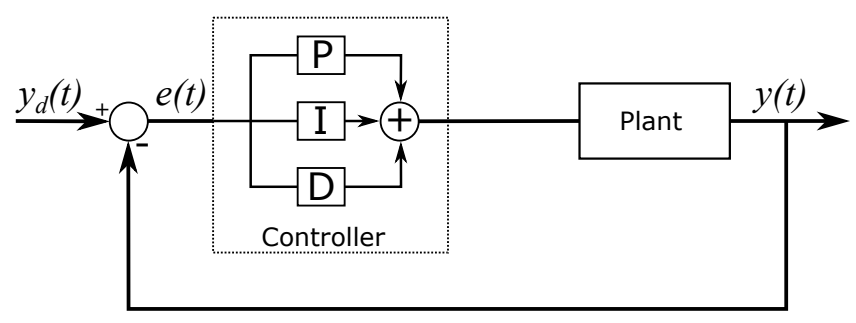

Figure 4: Schematic of a PID controller

An excellent introduction to the idea of using control theory for digital health interventions is provided by Rivera [43]. This gives a list of the required and desirable attributes for a problem to be suited to using control theory (Fig. 5). In addition to these considerations, applying control theory in non-standard applications can bring other challenges [57]: Measurements are likely to be highly noisy, and data may be sparse, missing or irregular. An even greater challenge is how we go about obtaining quantifiable measures for states and inputs, which may be subjective or not well defined.

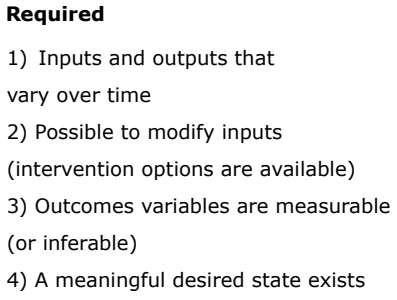

Figure 5: Attributes of a problem that are well matched to control engineering. Adapted from [43]

If these challenges can be overcome, control theory can provide a framework for determining optimal inputs. It inherently allows for individualisation via data-driven optimisation. To illustrate this we return to the PID feedback controller. The output signal is fed back and compared to a reference signal to generate an error signal, the inputs applied to the system are then determined from this error signal. In effect the inputs vary dependent on what the outputs are. When applied to an individual we effectively have an N-of-1 trial, as each set of inputs will differ, dependent on how the system responds. To further personalise digital health interventions there is the option of using adaptive control, where the parameters of the controller are adapted according to variations in the plant. These features have motivated research into applying control theory in medical treatments and to determine individualised treatments [8].

In addition to controlling the response of the system, control theory also enables us to study other aspects of the system such as the controllability and observability. The concept of controllability refers to the ability to manipulate a system using admissible inputs. In particular, the ability of an external input to move the internal state of a system $(\boldsymbol{x})$ from any initial state to any other final state [36]. The concept of observability refers to the ability to see what is going on inside the system and in particular how well the internal states of a system can be inferred from knowledge of its external outputs [36]. For a dynamical system model, the controllability and observability can be calculated from the state matrices $\boldsymbol{A}, \boldsymbol{B}, \boldsymbol{C}$. There is the potential that certain behaviours are not observable or controllable. This would be 
an interesting question to ask, but first requires a dynamical model.

\section{FUTURE CHALLENGES}

Using systems and control theory provides an exciting avenue to providing a theoretical basis for persuasive health. However, it also poses several questions and challenges that need addressing. To utilise systems and control theory we need to quantify inputs (including any constraints), outputs and states over time. Although some behavioural related measures are simple to quantify, such as the number and timing of messages (cues to action). Other measures, such as the content of the messages are more subjective and harder to quantify. Only simple, easy to quantify variables have been included in current dynamic systems models, or associated control based interventions. Key questions need to be addressed as to how these more qualitative measures can be quantified.

Control theory offers a framework for optimising inputs to ensure that a system behaves in a certain way. However, in the field of persuasion, we are unlikely to have complete control over these inputs. For example, we could send a message to motivate physical activity (cue to action), but we have no way of guaranteeing when the message will be read. This is not a common problem in control systems for engineering applications, which tend to have guaranteed inputs. Similar problems could arise in network control systems (e.g. delay, packet loss), however, there are usually some bounds on the delay [16]. Robustness to the fact that inputs cannot be guaranteed needs to be built into the system.

Of course, when dealing with persuasion we also need to consider ethics. There is a growing body of evidence that shows algorithms alone do not automatically treat diverse populations fairly [38]. Therefore, any control theory based algorithms need to provide methodologies for including ethics; these do not currently exist. Within a MPC framework there are methods to constrain inputs, which could be utilised to ensure unethical inputs are not used, but again we run into the problem of how to represent complex qualitative inputs numerically. Or, perhaps even more fundamentally the question of if we should be attempting to quantify these values. As well as a focus on developing a framework for persuasion, there needs to be a focus on addressing ethical questions. As well as those mentioned, there are also more generally applicable problems of data and algorithmic bias, misuse, liability, and unintended side effects of a successful persuasion.

Even once we have dynamic behaviour models and relevant controllers, we still need to ensure these can be utilised by computer scientist and developers to produce better interventions. We need something like the Action Behaviour Model [26], but for these dynamical models. This requires control systems engineers, behavioural scientists, computer scientists, and developers to work together to drive the field forward and come up with theory based persuasive technologies that will improve behaviours, and in turn health.

\section{CONCLUSIONS}

This paper has considered how ideas from systems and control theory could provide a theoretical framework that is currently lacking from persuasive technologies. It has reviewed the state of the art in the area and highlighted problems that need to be addressed in order to achieve this. We hope to have motivated the need for theoretical frameworks and better data sets, and installed the importance of capturing time in terms of dynamic behavioural changes.

\section{REFERENCES}

[1] Albert Bandura. 1977. Self-efficacy: Toward a Unifying Theory of Behavioral Change. Psychological Review 84, 2 (1977), 191-215. https: //doi.org/10.1037/0033-295X.84.2.191

[2] Marshall H Becker. 1974. The health belief model and personal health behavior. Health education monographs 2 (1974), 324-508. https: //doi.org/10.1177/109019817400200407

[3] Korkut Bekiroglu, Constantino Lagoa, Suzan A Murphy, and Stephanie T Lanza. 2017. Control engineering methods for the design of robust behavioral treatments. IEEE Transactions on Control Systems Technology 25, 3 (2017), 979-990. https://doi.org/10.1109/TCST.2016. 2580661

[4] Korkut Bekiroglu, Michael A. Russell, Constantino M. Lagoa, Stephanie T. Lanza, and Megan E. Piper. 2017. Evaluating the effect of smoking cessation treatment on a complex dynamical system. Drug and Alcohol Dependence 180, August (2017), 215-222. https://doi.org/10.1016/j.drugalcdep.2017.07.037

[5] B.C. Booske, J.K. Athens, D.A. Kindig, H. Park, and P.L. Remington. 2010. Different perspectives for assigning weights to determinants of health. University of Wisconsin: Population Health Institute.

[6] Belinda Borrelli and Lee M Ritterband. 2015. Special issue on eHealth and mHealth: Challenges and future directions for assessment, treatment, and dissemination. Health Psychology 34, S (2015), 1205-1208. https://doi.org/10.1037/hea0000323

[7] Ankush Chakrabarty, Stamatina Zavitsanou, Francis J Doyle, and Eyal Dassau. 2018. Event-triggered model predictive control for embedded artificial pancreas systems. IEEE Transactions on Biomedical Engineering 65, 3 (2018), 575-586.

[8] Q. Clairon, E.D. Wilson, R. Henderson, and C.J. Taylor. 2017. Adaptive Biomedical Treatment and Robust Control. IFAC-PapersOnLine 50, 1 (2017), 12191-12196. https://doi.org/10.1016/j.ifacol.2017.08.2274

[9] Hein de Vries. 2017. An Integrated Approach for Understanding Health Behavior; The I-Change Model as an Example. Psychology and Behavioral Science International fournal 2, 2 (2017). https: //doi.org/10.19080/pbsij.2017.02.555585

[10] Sunil Deshpande, Naresh N Nandola, Daniel E Rivera, and Jarred W Younger. 2014. Optimized treatment of fibromyalgia using system identification and hybrid model predictive control. Control engineering practice 33 (2014), 161-173. https://doi.org/10.1016/j.conengprac.2014. 09.011

[11] Yuwen Dong, Sunil Deshpande, Daniel E Rivera, Danielle S Downs, and Jennifer S Savage. 2014. Hybrid model predictive control for sequential decision policies in adaptive behavioral interventions. In 
PervasiveHealth'19, May 20-23, 2019, Trento, Italy Emma D. Wilson, Sumi Helal, Christopher N. Bull, and Mahsa Honary

2014 American Control Conference. IEEE, 4198-4203. https://doi.org/ 10.1016/j.conengprac.2014.09.011

[12] Yuwen Dong, Daniel E Rivera, Danielle S Downs, Jennifer S Savage, Diana M Thomas, and Linda M Collins. 2013. Hybrid model predictive control for optimizing gestational weight gain behavioral interventions. In 2013 American Control Conference. IEEE, 1970-1975.

[13] Yuwen Dong, Daniel E. Rivera, Diana M. Thomas, Jesus E. NavarroBarrientos, Danielle S. Downs, Jennifer S. Savage, and Linda M. Collins. 2012. A Dynamical Systems Model for Improving Gestational Weight Gain Behavioral Interventions. Proc Am Control Conf. (2012), 40594064.

[14] B.J. Fogg. 2003. Persuasive Technology: Using Computers to Change What We Think and Do. Morgan Kaufmann. 1-282 pages.

[15] Mohammad T. Freigoun, Cesar A. Martin, Daniel E. Rivera, Teresa P. Azevedo Perdicoulis, Paulo Lopes dos Santos, Rodrigo Alvite Romano, and Eric B. Hekler. 2018. System Identification of Just Walk: Using Matchable-Observable Linear Parametrizations. IEEE Transactions on Control Systems Technology (2018), 1-12. https://doi.org/10.1109/TCST. 2018.2884833

[16] R. A. Gupta and M. Chow. 2010. Networked Control System: Overview and Research Trends. IEEE Transactions on Industrial Electronics 57, 7 (2010), 2527-2535. https://doi.org/10.1109/TIE.2009.2035462

[17] Juho Hamari, Jonna Koivisto, and Tuomas Pakkanen. 2014. Do persuasive technologies persuade?-a review of empirical studies. In In: Spagnolli A., Chittaro L., Gamberini L. (eds) Persuasive Technology. PERSUASIVE 2014. Lecture Notes in Computer Science, Vol. 8462. Springer, 118-136. https://doi.org/10.1007/978-3-319-07127-5_11

[18] Erik B Hekler, Predrag Klasnja, Vicente Traver, and Monique Hendriks. 2013. Realizing effective behavioral management of health: the metamorphosis of behavioral science methods. IEEE pulse 4, 5 (2013), 29-34.

[19] Eric B Hekler, Susan Michie, Misha Pavel, Daniel E Rivera, Linda M Collins, Holly B Jimison, Claire Garnett, Skye Parral, and Donna Spruijt-Metz. 2016. Advancing models and theories for digital behavior change interventions. American journal of preventive medicine 51, 5 (2016), 825-832

[20] K. Johan Åström and R.M. Murray. 2010. Feedback systems: An introduction for scientists and engineers. Princeton University Press.

[21] Robert M Kaplan and Arthur A Stone. 2013. Bringing the laboratory and clinic to the community: mobile technologies for health promotion and disease prevention. Annual review of psychology 64 (2013), 471498.

[22] Michael P. Kelly and Mary Barker. 2016. Why is changing healthrelated behaviour so difficult? Public Health 136 (2016), 109-116. https: //doi.org/10.1016/j.puhe.2016.03.030

[23] Predrag Klasnja, Eric B. Hekler, Saul Shiffman, Audrey Boruvka, Daniel Almirall, Ambuj Tewari, and Susan A. Murphy. 2015. MicroRandomized Trials: An Experimental Design for Developing Just-inTime Adaptive Interventions. Health Psychol 34, 34 (2015), 1220-1228. https://doi.org/10.1037/hea0000305

[24] Alexis Kuerbis, Christine Davis, Karyn L. Sutton, Keri L. Rehm, Jon Morgenstern, H. T. Banks, and Lisa Hail. 2014. Dynamic modeling of behavior change. Quart. Appl. Math. 72, 2 (2014), 209-251. https: //doi.org/10.1090/s0033-569x-2014-01296-3

[25] Peter J. Lawson and Susan A. Flocke. 2009. Teachable moments for health behaviour change: a concept analysis. Patient Educ Couns., 76, 1 (2009), 25-30. https://doi.org/10.1016/j.pec.2008.11.002

[26] Duckki Lee, Abdelsalam (Sumi) Helal, Yunsick Sung, and Stephen Anton. 2015. Situation-Based Assess Tree for User Behavior Assessment in Persuasive Telehealth. IEEE Transactions on Human-Machine Systems 45, 5 (2015), 624-634. https://doi.org/10.1109/THMS.2015.2443712
[27] Duckki Lee, Sumi Helal, Steve Anton, Scott De Deugd, and Andy Smith. 2012. Participatory and persuasive telehealth. Gerontology 58, 3 (2012), 269-281.

[28] Duckki Lee, Sumi Helal, and Brian David Johnson. 2010. An actionbased behavior model for persuasive telehealth. In In: Lee Y. et al. (eds) Aging Friendly Technology for Health and Independence. ICOST 2010. Lecture Notes in Computer Science. Springer, 121-129. https: //doi.org/10.1007/978-3-642-13778-5_15

[29] Douglas G MacMartin, Ben Kravitz, David W Keith, and Andrew Jarvis. 2014. Dynamics of the coupled human-climate system resulting from closed-loop control of solar geoengineering. Climate dynamics 43, 1-2 (2014), 243-258. https://doi.org/10.1007/s00382-013-1822-9

[30] Cesar A. Martin, Daniel E. Rivera, Eric B. Hekler, William T. Riley, Matthew P. Buman, Marc A. Adams, and Alicia B. Magann. 2018. Development of a Control-Oriented Model of Social Cognitive Theory for Optimized mHealth Behavioral Interventions. IEEE Transactions on Control Systems Technology (2018), 1-16. https://doi.org/10.1109/ TCST.2018.2873538

[31] SF Michie, Robert West, Rona Campbell, Jamie Brown, and Heather Gainforth. 2014. ABC of behaviour change theories. Silverback Publishing.

[32] Arlen C Moller, Gina Merchant, David E Conroy, Robert West, Eric Hekler, Kari C Kugler, and Susan Michie. 2017. Applying and advancing behavior change theories and techniques in the context of a digital health revolution: proposals for more effectively realizing untapped potential. Journal of behavioral medicine 40, 1 (2017), 85-98.

[33] Inbal Nahum-Shani, Eric B Hekler, and Donna Spruijt-Metz. 2015. Building health behavior models to guide the development of just-intime adaptive interventions: A pragmatic framework. Health Psychology 34, S (2015), 1209.

[34] J.-Emeterio Navarro-Barrientos, Daniel E. Rivera, and Linda M. Collins. 2011. A dynamical model for describing behavioural interventions for weight loss and body composition change. Mathematical and Computer Modelling of Dynamical Systems 17, 2 (2011), 183-203.

[35] J.W. Nicklow. 2000. Discrete-time optimal control for water resources engineering and management. Water International 25, 1 (2000), 89-95. https://doi.org/10.1080/02508060008686800

[36] Katsuhiko Ogata. 2010. Modern Control Engineering (5th ed.). Prentice Hall.

[37] Rita Orji and Karyn Moffatt. 2018. Persuasive technology for health and wellness: State-of-the-art and emerging trends. Health informatics journal 24, 1 (2018), 66-91.

[38] Osonde A. Osoba and William Welser. 2017. An intelligence in our image: The risks of bias and errors in artificial intelligence. (2017).

[39] Andrew Prestwich, Falko F Sniehotta, Craig Whittington, Stephan U Dombrowski, Lizzie Rogers, and Susan Michie. 2014. Does theory influence the effectiveness of health behavior interventions? Metaanalysis. Health Psychology 33, 5 (2014), 465.

[40] James O Prochaska and Wayne F Velicer. 1997. The transtheoretical model of health behavior change. American journal of health promotion 12, 1 (1997), 38-48.

[41] William T Riley, Daniel E Rivera, Audie A Atienza, Wendy Nilsen, Susannah M Allison, and Robin Mermelstein. 2011. Health behavior models in the age of mobile interventions: are our theories up to the task? Translational behavioral medicine 1, 1 (2011), 53-71.

[42] Daniel E. Rivera. 2012. Optimized behavioral interventions: What does system identification and control engineering have to offer? Vol. 16. IFAC. 882-893 pages. https://doi.org/10.3182/20120711-3-BE-2027.00427 
[43] Daniel E Rivera, Matthew P Buman, Cesar A Martin, Mohammad T Freigoun, Eric B Hekler, Marc A Adams, Elizabeth Korinek, Sayali S Phatak, and Predrag Klasnja. 2018. Tutorial for Using Control Systems Engineering to Optimize Adaptive Mobile Health Interventions. Journal of Medical Internet Research 20, 6 (2018), e214. https://doi.org/10.2196/jmir.8622

[44] Daniel E Rivera, Michael D Pew, and Linda M Collins. 2008. Using Engineering Control Principles to Inform the Design of Adaptive Interventions: A Conceptual Introduction. Drug Alcohol Dedend. 88, Suppl 2 (2008), 1-23.

[45] Niilo Saranummi, Donna Spruijt-Metz, Stephen Intille, Llkka Korhonen, Wendy J. Nilsen, and Misha Pavel. 2016. Moving the Science of Behavioral Change into the 21st Century. IEEE Pulse 4, 5 (2016), 22-24. https://doi.org/10.1109/MPUL.2013.2271680

[46] Ralf Schwarzer. 2008. Modeling health behavior change: How to predict and modify the adoption and maintenance of health behaviors. Applied Psychology 57, 1 (2008), 1-29. https://doi.org/10.1111/j.1464-0597.2007. 00325.x

[47] Suresh P. Sethi and Gerald L. Thompson. 2000. Optimal Control Theory: Applications to Management Science and Economics. Springer.

[48] Saul Shiffman, Arthur A Stone, and Michael R Hufford. 2008. Ecological momentary assessment. Annu. Rev. Clin. Psychol. 4 (2008), 1-32.

[49] Joshua Smyth, Korkut Bekiroglu, Stephanie Lanza, David Conroy, Mahmoud Ashour, Chih-Hsiang Yang, and Constantino Lagoa. 2016. On the mathematical modeling of the effect of treatment on human physical activity. 2016 IEEE Conference on Control Applications (CCA) (2016), 1084-1091. https://doi.org/10.1109/cca.2016.7587951

[50] Joshua M. Smyth, Chih-Hsiang Yang, Sarah Hojjatinia, Stephanie T. Lanza, Constantino M. Lagoa, and David E. Conroy. 2018. Personalized models of physical activity responses to text message microinterventions: A proof-of-concept application of control systems engineering methods. Psychology of Sport and Exercise 41, June 2018 (2018), 172-180. https://doi.org/10.1016/j.psychsport.2018.06.011

[51] Donna Spruijt-Metz, Eric Hekler, Niilo Saranummi, Stephen Intille, Ilkka Korhonen, Wendy Nilsen, Daniel E Rivera, Bonnie Spring, Susan Michie, David A Asch, et al. 2015. Building new computational models to support health behavior change and maintenance: new opportunities in behavioral research. Translational behavioral medicine 5, 3 (2015), 335-346.

[52] Donna Spruijt-Metz, Eric Hekler, Niilo Saranummi, Stephen Intille, Ilkka Korhonen, Wendy Nilsen, Daniel E. Rivera, Bonnie Spring, Susan Michie, David A. Asch, Alberto Sanna, Vicente Traver Salcedo, Rita Kukakfa, and Misha Pavel. 2015. Building new computational models to support health behavior change and maintenance: new opportunities in behavioral research. Translational Behavioral Medicine 5, 3 (2015), 335-346. https://doi.org/10.1007/s13142-015-0324-1

[53] Shane N Sweet and Michelle S Fortier. 2010. Improving physical activity and dietary behaviours with single or multiple health behaviour interventions? A synthesis of meta-analyses and reviews. International journal of environmental research and public health 7, 4 (2010), 1720-1743.

[54] C.J. Taylor and J.-M. Aerts. 2014. Control of Nonlinear Biological Systems by Non-minimal State Variable Feedback. Statistics in Biosciences 6, 2 (2014), 290-313. https://doi.org/10.1007/s12561-013-9098-5

[55] Kevin P Timms, Daniel E Rivera, Megan E Piper, and Linda M Collins. 2014. A hybrid model predictive control strategy for optimizing a smoking cessation intervention. In 2014 American Control Conference. IEEE, 2389-2394.

[56] Corneel Vandelanotte, Andre M Müller, Camille E Short, Melanie Hingle, Nicole Nathan, Susan L Williams, Michael L Lopez, Sanjoti Parekh, and Carol A Maher. 2016. Past, present, and future of eHealth and mHealth research to improve physical activity and dietary behaviors. fournal of nutrition education and behavior 48, 3 (2016), 219-228.

[57] Emma D Wilson, Quentin Clairon, Robin Henderson, and C James Taylor. 2018. Dealing with observational data in control. Annual Reviews in Control (2018).

[58] P. C. Young and H. Garnier. 2006. Identification and estimation of continuous-time, data-based mechanistic (DBM) models for environmental systems. Environmental Modelling and Software 21, 8 (2006), 1055-1072. 